\title{
Qubit-photon corner states in all dimensions
}

\author{
Adrian Feiguin, ${ }^{1}$ Juan José García-Ripoll, ${ }^{2}$ and Alejandro González-Tudela $\odot^{2}$ \\ ${ }^{1}$ Department of Physics, Northeastern University, Boston, Massachusetts 02115, USA \\ ${ }^{2}$ Institute of Fundamental Physics IFF-CSIC, Calle Serrano 113b, 28006 Madrid, Spain
}

(Received 22 October 2019; accepted 30 March 2020; published 27 April 2020)

\begin{abstract}
A single quantum emitter coupled to a one-dimensional photon field can perfectly trap a photon when placed close to a mirror. This occurs when the interference between the emitted and reflected light is completely destructive, leading to photon confinement between the emitter and the mirror. In higher dimensions, the spread of the light field in all directions hinders interference and, consequently, photon trapping by a single emitter remains elusive so far. In this work, we show how a single emitter can indeed trap light in any dimension. We provide a constructive recipe based on judiciously coupling an emitter to a photonic crystal-like bath with properly designed open boundary conditions. The directional propagation of the photons in such baths enables perfect destructive interference, forming what we denote as qubit-photon corner states. We characterize these states in all dimensions, showing that they are robust under fluctuations of the emitter's properties, and persist also in the ultrastrong coupling regime.
\end{abstract}

DOI: 10.1103/PhysRevResearch.2.023082

\section{INTRODUCTION}

The radiation properties of a quantum emitter can change, modifying the photonic environment around it [1]. A particularly simple example of this consists in placing an emitter close to a mirror [2-4] or to other quantum emitters [5,6]. These configurations in free space already lead to remarkable effects, such as lifetime renormalizations or the modification of atomic resonance fluorescence [7-11]. However, they are ultimately limited by the reduced solid angle of the emitted light that the mirrors or emitters can cover. All these effects are dramatically enhanced when the emitters couple to onedimensional (1D) photonic fields such as dielectric [12-17] or microwave [18] waveguides, where, for example, a single atom can perfectly reflect single photons [19]. These strong interference effects can lead to the emergence of bound states in the continuum (BIC) with two emitters [20-27], or a single emitter in front of a mirror [28-33], in which a single photon becomes localized despite being energetically in the middle of the continuous spectrum. These counterintuitive states, originally proposed by von Neumann and Wigner in a different context [34], have experienced a renewed interest due to their experimental demonstration in several classical systems, such as photonic crystals [35] or acoustic waves [36,37], and their possible applications in lasing or sensing (see Ref. [38] and references therein). BICs formed by quantum emitters, differently from the classical ones, are entangled light-matter states that can be used for quantum information purposes, for example, in the design of decoherence-free quantum gates

Published by the American Physical Society under the terms of the Creative Commons Attribution 4.0 International license. Further distribution of this work must maintain attribution to the author(s) and the published article's title, journal citation, and DOI.
$[39,40]$ or nonreciprocal photon transport [41-43]. Since such BICs will be the focus of this article, we will use the label qBIC to distinguish them from their classical counterparts.

Among the different configurations, the one using a single emitter and a mirror [28-33] is especially advantageous, since the qBICs in that case are insensitive to the energy mismatch between emitters. Exporting this configuration to higherdimensional systems is challenging because the wave-packet diffraction precludes in general perfect destructive interference, that is, that the reflected photons arrive with the same amplitude to the emitter but out of phase. Here we provide an example where a single quantum emitter can perfectly trap light and create a (infinite lifetime) qBIC in any dimension. The key idea is to combine the directional emission occurring in two- and three-dimensional (2D and 3D) photonic crystallike baths [44-47], with an adequate design of open boundary conditions. Then, by placing the emitter close to a corner of the photonic bath, its directional emission and the reflection in the boundary generates a high-dimensional qBIC that we label as a qubit-photon corner state. In contrast to the recently observed topological photon corner states [48-55], ours can inherit a strong nonlinearity from the emitter and do not require a topologically nontrivial bath. We characterize these states in one, two, and three dimensions using exact numerical techniques to take into account the retardation effects and the corrections in the ultrastrong coupling regime, where these states acquire a finite lifetime even in lossless baths.

\section{SETUP}

To illustrate the emergence of these states, we use a $d$ dimensional photonic lattice composed by $N^{d}$ resonators with energy $\omega_{a}$ that can tunnel to their nearest neighbor at a rate $J$. With these assumptions, the bath energy dispersion $(\omega(\mathbf{k}))$ then depends only on the photonic lattice geometry, which determines the number of nearest-neighbor resonators $\left(N_{n n}\right)$. 
For the emitter, we take a two-level system (qubit) with energy difference $\Delta$ that is locally coupled at a position $\mathbf{x}_{0} \in \mathbf{R}^{d}$ to the photonic bath. Thus, the full Hamiltonian reads

$$
H=\frac{\Delta}{2} \sigma^{z}+\sum_{\mathbf{x}} \omega_{a} a_{\mathbf{x}}^{\dagger} a_{\mathbf{x}}+J \sum_{\langle\mathbf{x}, \mathbf{y}\rangle} a_{\mathbf{x}}^{\dagger} a_{\mathbf{y}}+g_{x_{0}} \sigma^{x}\left(a_{\mathbf{x}_{0}}+a_{\mathbf{x}_{0}}^{\dagger}\right)
$$

Notice that we have kept the full dipole coupling $g_{\mathbf{x}_{0}}$ between the emitter and the photonic mode. In this way we can study situations in which the coupling is comparatively weak $g \ll \Delta, \omega_{a}$ and the rotating-wave approximation (RWA) is justified, replacing $g \sigma^{x}\left(a+a^{\dagger}\right) \sim g\left(\sigma^{+} a+\sigma^{-} a^{\dagger}\right)$, but also the ultrastrong coupling (USC) regime, which occurs when $g / \Delta \geqslant 10 \%$. In this limit, the physics of the emitter changes substantially when $g \sim W$, where $W=2 N_{n n} J$ is the photon bandwidth.

We are interested in studying the spontaneous emission dynamics, that is, considering that the emitter is initially excited with no photons in the bath, and then study the time dynamics governed by $e^{-i H t}$. In our case, this is a complicated problem because of the high-dimensional nature of the bath and, in the ultrastrong coupling regime, because the number of excitations is not conserved. Thus, before describing the physics, it is worth explaining the two complementary approaches we used to study this problem [56].

\section{A. Polaron Hamiltonian}

Instead of working with (1) directly, we study the unitarily equivalent polaron Hamiltonian [57]. This transformed model eliminates much of the entanglement between the quantum emitter and the photonic field, leading to renormalized coupling strengths and qubit frequencies. For moderate coupling strengths or finite-bandwidth models, the polaron Hamiltonian has a single-excitation limit that describes the spontaneous emission problem that we want to study:

$$
\begin{aligned}
H_{\mathrm{pol}, 1 \mathrm{D}}= & \frac{\tilde{\Delta}}{2} \sigma^{z}\left(1+8 F^{\dagger} F\right)+\sum_{\mathbf{x}, \mathbf{y}} J_{\mathbf{x y}} a_{\mathbf{x}}^{\dagger} a_{\mathbf{y}} \\
& +2 \tilde{\Delta}\left(\sigma^{+} F+\text { H.c. }\right)+\sum_{\mathbf{x}} \omega_{a} a_{\mathbf{x}}^{\dagger} a_{\mathbf{x}} .
\end{aligned}
$$

Within that picture, the emitter interacts with a collective coupling operator $F=\sum_{\mathbf{x}} f_{\mathbf{x}} a_{\mathbf{x}}$ with coupling vector $\mathbf{f}=$ $\left\{f_{\mathbf{x}}\right\}_{\mathbf{x}}$, and has a renormalized frequency $\tilde{\Delta}$. These parameters can be obtained solving self-consistently the following equations:

$$
\tilde{\Delta}=\Delta e^{-2 \sum_{\mathbf{k}}\left|f_{\mathbf{k}}\right|^{2}}, \quad \mathbf{f}=\frac{1}{J+\tilde{\Delta}} \mathbf{g},
$$

where $f_{\mathbf{k}}$ is the Fourier transform of $\mathbf{f}$, and $\mathbf{g}$ is the original coupling vector of the emitter-bath Hamiltonian in real space. The single-excitation polaron adopts a RWA stanza and is therefore amenable to analytical treatment, much like earlier works with regular lattices and pointlike interactions $[44,45]$. As a result, the model supports single-excitation solutions,

$$
|\psi(t)\rangle=\left[\sum_{\mathbf{x}} \psi(\mathbf{x}, t) a_{\mathbf{x}}^{\dagger}+c(t) \sigma^{+}\right]|\downarrow\rangle \otimes|\mathrm{vac}\rangle,
$$

$|v a c\rangle$ being the bath state with no photon, and whose photon and qubit components $\psi(\mathbf{x}, t)$ and $c(t)$ follow a linear Schrödinger equation with $H_{\mathrm{pol}, 1 \mathrm{D}}$, that can be evolved in time using different numerical methods. Note that as long as $\psi(\mathbf{x}, t), c(t) \neq 0$, this represents an entangled state between the qubit and photonic component.

\section{B. Chain mapping and DMRG}

As an additional benchmark, we also solve the dynamics of the full spin-boson model of Eq. (1) using a time-dependent version of the density matrix renormalization group (tDMRG) [58-61]. To simulate large high-dimensional bosonic baths, the noninteracting lattice Hamiltonian is exactly mapped (it is a unitary transformation) onto a $1 \mathrm{D}$ chain of free bosons by means of a Lanczos recursion [62-64]. The consequent dimensional and entanglement reduction makes the new Hamiltonian amenable to DMRG simulations. Remarkably, with only $N$ bosonic modes in the chain we capture well the dynamics of the emitter. As shown in [65], this mapping can be combined with the polaron transformation to reduce the amount of entanglement, but this was not required for this study.

\section{REMINDER OF $1 D$ qBICS}

Our first set of simulations recreates the BICs obtained in a one-dimensional lattice with open boundaries and $N=400$ sites, taking the lattice constant as the unit of length [see Fig. 1(a)]. We use an emitter resonant with the middle of the photonic band, $\Delta=\omega_{a}=2.5 \mathrm{~J}$, although this is not strictly needed in the $1 \mathrm{D}$ case. We place a quantum emitter at even ( $x=12$, solid) and odd positions ( $x=11$, dashed), excite the emitter, and abruptly switch on the coupling $g$. When the emitter is placed on an odd site, it decays completely, releasing a propagating photon. However, if the emitter is on an even site, it can, with some probability, trap a photon between the emitter and the end of the lattice, as seen in Fig. 1(a). The reason for the difference between the even and odd sites is that when $\Delta=\omega_{a}$, the momentum of the resonant photons is $k_{0}=\pi / 2$, such that the phase acquired by them after reflecting with the mirror reads $-e^{i 2 k_{0} x}=(-1)^{x+1}$, because it travels a distance $2 x$ and acquires an extra $\pi$ phase with the reflection of the mirror. Thus, for even sites the reflected photon arrives out-of-phase to the emitter, leading to a perfect destructive interference with the photons that it emits and, consequently, becomes trapped between the emitter and the mirror.

Such states at even sites correspond to the qBICs that have been identified before in one-dimensional systems [20-33] and can be also intuitively understood from the interference between the emitted light of the emitter and its afterimage, as schematically depicted in Fig. 1(a). Figure 1(b) plots the probability of creating the $1 \mathrm{D}$ qBIC, defined as

$$
N_{\text {excit }}=\frac{1}{2}\left(\sigma^{z}+1\right)+\sum_{x=1}^{x_{0}} a_{x}^{\dagger} a_{x}=P_{\uparrow}+P_{\gamma},
$$

which contains both a non-negligible photonic $\left(P_{\gamma}\right)$ and qubit $\left(P_{\uparrow}\right)$ component. Note how the emitter in even sites have some probability to excite the qBIC, even in the USC regime. As 
we increase the coupling strength, the qBIC transitions from being mostly an excited atom to an equal superposition of both [cf. Fig. 1(c)]. In the USC regime, the qBIC state has a significant fraction of photon component, and it also acquires a finite lifetime [cf. Fig. 1(d)]. This lifetime can be attributed to the deviation of the perfect interference condition due to the renormalization of the qubit energy when $g \sim W$, since $\tilde{\Delta} \neq \Delta$ [see Eq. (3)]. This renormalized energy makes the momentum of the resonant photons different such that the photons will not arrive perfectly out-of-phase to the emitter position. Although not shown explicitly, the results obtained using the single-photon polaron Hamiltonian agree very well with our DMRG simulations.

(a) Afterimage $\sim \pi$-shift reflection
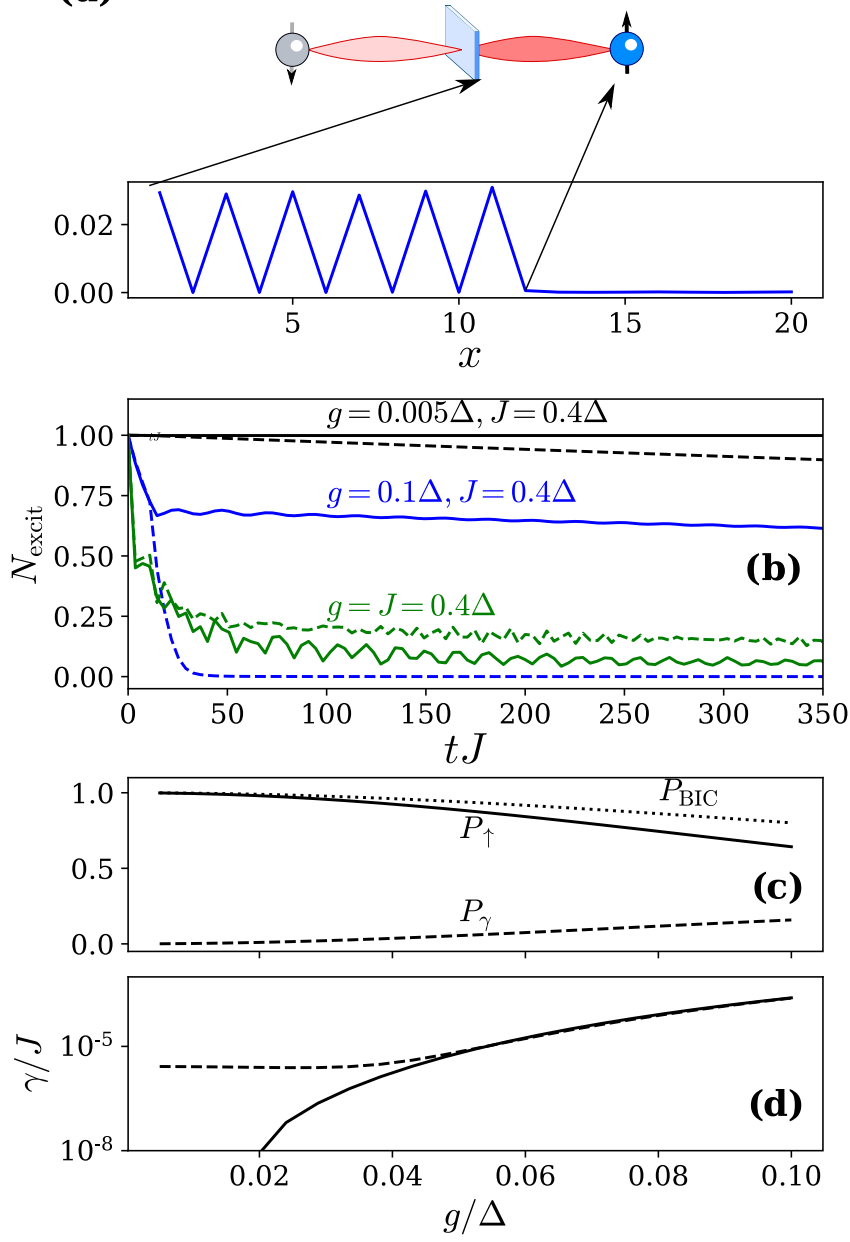

FIG. 1. Formation of a $1 \mathrm{D}$ qBIC by spontaneous emission on a 1D lattice with 400 sites. (a) Pictorial representation (above) and photon number spatial distribution (below) in the BIC state for $g=$ $0.1 \Delta$ and $x=12$. (b) Total excitation number $N_{\text {excit }}(5)$ in the qBIC state as a function of time. Solid and dashed lines correspond to the $x=12$ th and $x=11$ th sites. (c) Qubit and photon component of the bound state, $P_{\uparrow}$ and $P_{\gamma}$, and probability of creating the bound state $P_{\mathrm{qBIC}} \sim N\left(t_{0}\right)$ for $t_{0} J=90$. (d) Estimated decay rate of the corner state extracted from a fit $N_{\text {excit }}(t) \sim N\left(t_{0}\right) \exp \left(-\gamma\left(t-t_{0}\right)\right)$ after the initial transient for a simulation time $t J \in(45,90){ }^{1}$

${ }^{1}$ Values below $10^{-5}$ are not reliable, due to finite simulation time. (a)
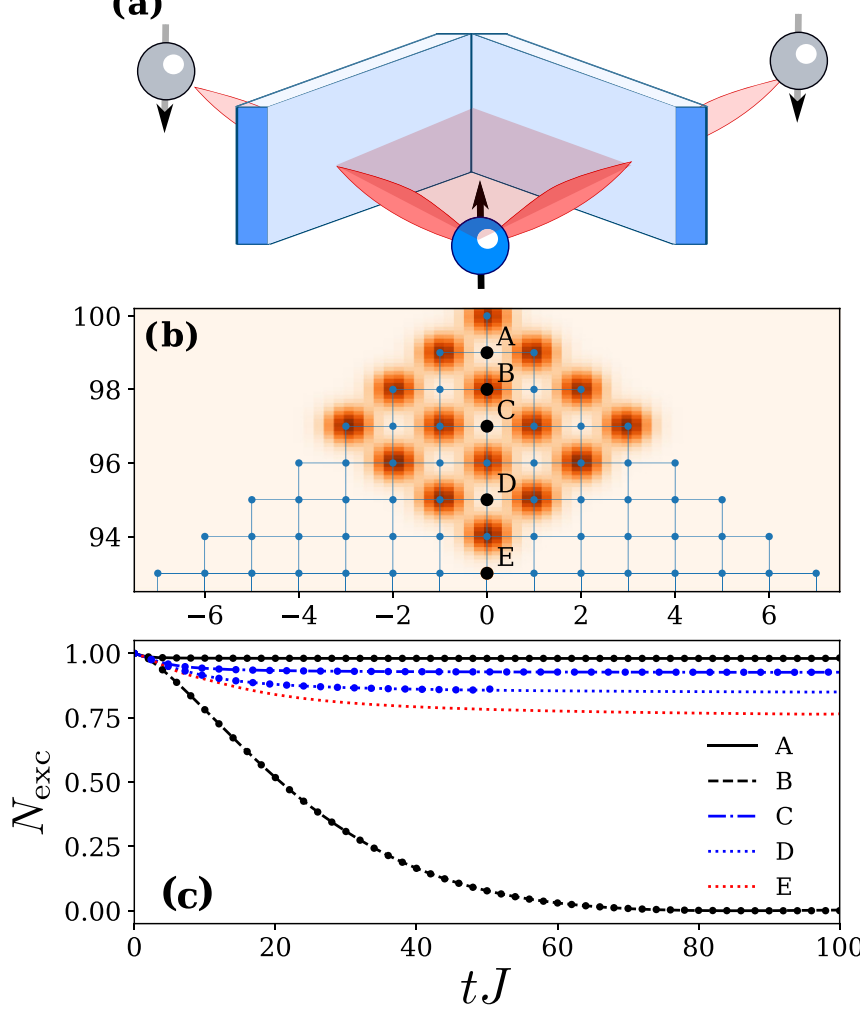

FIG. 2. Formation of a corner state by spontaneous emission on a $2 \mathrm{D}$ rhombic lattice with 30 sites on each diagonal for $g=$ $0.01 \Delta$ and $J=0.4 \Delta$. (a) Pictorial representation of the emitter and its afterimages. (b) Locations of the emitter in the corner of the photonic lattice (dots), coupling between photonic sites (lines), and distribution of photons (density plot) for a corner state generated by emitter $E$. (c) Total excitation number $N_{\text {excit }}$ as a function of time, for different locations of the emitter, from $A$ to $E$.

\section{QUBIT-PHOTON CORNER STATES IN 2D}

To obtain these phenomena in two dimensions, it is enough to consider the simpler generalization of the coupled cavity array to two dimensions, that is, disposing the resonators in a square geometry. This model displays an energy dispersion given by

$$
\omega_{2 \mathrm{D}}(\mathbf{k})=\omega_{a}+2 J\left[\cos \left(k_{x}\right)+\cos \left(k_{y}\right)\right] .
$$

At the middle of the band, $\omega(\mathbf{k})=\omega_{a}$, the isofrequencies are "nested," which means they are straight lines defined by $k_{x} \pm k_{y}= \pm, \mp \pi$. Note, these lines include $\mathbf{k}$ modes with maximal group velocity, $( \pm \pi / 2, \mp \pi / 2)$, but others with strictly zero group velocity, $(0( \pm \pi), \pm \pi(0))$. One of the consequences of such inhomogeneity is that when an emitter is spectrally tuned to that energy, its emission becomes highly directional [44-46]. This is what we will harness to induce the perfect trapping. The key idea consists in designing the boundaries around the emitter in a way that they are orthogonal to the preferential emission directions of the emitter [see Fig. 2(a)]. The intuition is then that the emitter's afterimages are out of phase with respect to the emission from the emitter. 
Figure 2(b) shows a proof-of-principle example of that mechanism. We have taken a square lattice and removed sites to form a reflective corner in a rhombus with $4 \times 30^{2}$ sites. The quantum emitter is equidistant to its afterimages only when placed on the diagonal of the rhombus, positions $A$ to $E$ in the plot. As in the 1D case, when we place the emitter on an odd site, such as B, it fails to acquire the right phase relation and decays, releasing a photon into the lattice. However, for even positions (A, C, D, E) the emitter relaxes to a stationary state with high probability [cf. Fig. 2(b)]. In these states, the photon is trapped in a corner, avoiding the quantum emitter. Figure 2(b) shows a density plot of a trapped photon that is anchored by a quantum emitter at position E. Remarkably, these photon corner states do not appear as a linear combinations of $1 \mathrm{D}$ bound states but rather occupy the whole area, since the emission occurs eventually in all directions. As in the $1 \mathrm{D}$ case, we have a very good agreement between DMRG and the single-photon polaron Hamiltonian for the rhombus. However, since the DMRG is working with a reduced number of modes (up to four per bath site), it allows the simulation of larger lattices-see Fig. 2(c), where the DMRG uses $400^{2}$ sites-and even moves to higher-dimensional scenarios, as we will show next.

\section{QUBIT-PHOTON CORNER STATES IN 3D}

In the three-dimensional case there are many different geometries in which the resonators can be disposed, but not all of them are suitable for our purposes. Using the intuition developed in Ref. [47], we choose a body-centered-cubic lattice in which each resonator is connected to eight nearest neighbors. This model has an energy dispersion,

$$
\begin{aligned}
\omega_{3 \mathrm{D}}(\mathbf{k})= & \omega_{a}+2 J\left[\cos \left(k_{x}\right)+\cos \left(k_{y}\right)\right. \\
& \left.+\cos \left(k_{z}\right)+\cos \left(k_{x}+k_{y}+k_{z}\right)\right],
\end{aligned}
$$

with nested equifrequencies that yield highly collimated emission in three directions. This is especially well suited to provide reflection in 3D corners. Other geometries, like the cubic-simple lattices, also display collimated emission but in more directions [47], such that they are not adequate for the desired goal.

In Fig. 3 we provide a proof-of-principle numerical confirmation of the trapping for a lattice with $N=120^{3}$ and $g / \Delta=0.1$. Figure 3(a) shows the 3D photon distribution of a qubit-photon corner state when placed in the position denoted by the red dot (D), while in Fig. 3(b) we plot an horizontal cut of this distribution. Finally, in Fig. 3(c) we plot the probability of exciting the BIC as a function of time for positions A-D depicted in Fig. 3(b) comparing again the polaron Hamiltonian (lines) and chain-mapped DMRG (dots). Here again, we see the difference between the positions A, C, and $\mathrm{D}$, where the phase relation with the afterimages is the right one, compared the $\mathrm{B}$ situation where the photon is not trapped, and qBIC probability is very small.

\section{IMPERFECTIONS}

So far, we have considered the ideal situation in which neither the qubit nor the cavity modes have intrinsic losses. However, photonic losses (at rates $\kappa_{\mathbf{x}}$ ) generally appear either

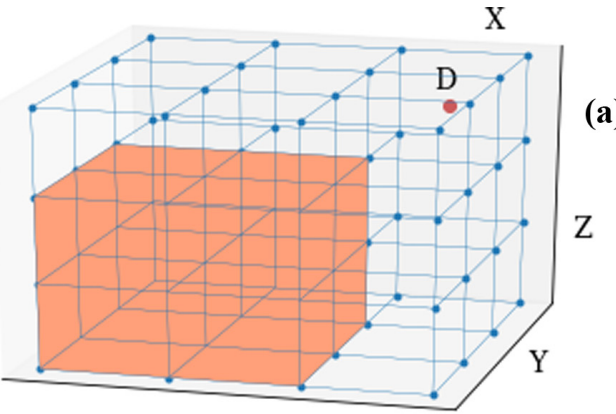

(a)
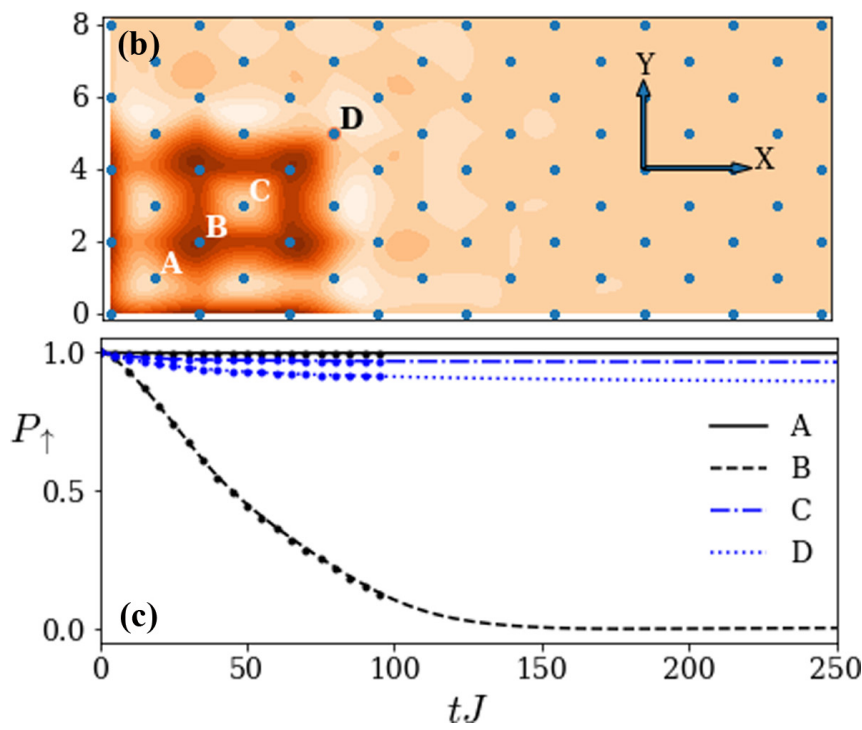

FIG. 3. (a) A cube of light in a corner state trapped by a quantum emitter at position $\mathrm{D}(x=y=z=5)$ on the diagonal of a bcc photonic lattice. (b) Density of photons on the corner state, as seen from above. (c) Probability of creating a corner state for emitters at A, B, C, and D (respectively $x=y=z \in\{1,2,3,5\}$ ), for $J=$ $0.4 \Delta, g=0.005 \Delta$ in Eq. (1). Dots are simulations using the DMRG algorithm with 400 Lanczos states.

because the reflecting boundary conditions are not perfect or because of absorption in the material. Besides, the qubit generally has an intrinsic finite lifetime $\left(\gamma_{\mathrm{qb}}\right)$ because of the coupling to different environments. The main consequence of these two imperfections is to provide to the qubit-photon corner state a finite lifetime $\gamma$, even in the rotating-wave situation where the qBIC lifetime should become infinite. Assuming a constant decay rate in all cavity modes $\kappa$, the lifetime introduced by these two mechanisms can be shown to be given by $\gamma=P_{\gamma} \kappa+\gamma_{\mathrm{qb}} P_{\uparrow}$. In the Markovian regime $(g \ll J)$, it can be shown that $P_{\uparrow} \sim 1$ and $P_{\gamma} \sim O\left(g^{2} / J^{2}\right)$, such that the lifetime will be dominated by the qubit losses.

\section{CONCLUSIONS}

Summing up, in this work we have shown that a single quantum emitter can trap a photon in any dimension. The emitter must be placed in a photonic crystal-like medium, with the right separation from the reflective boundaries of the medium. Under such conditions, the emitter interferes destructively with the afterimages reflected by the boundaries, generating a bound state in the continuum that we denote as a qubit-photon corner state. We have shown evidence 
of this effect in 1D, 2D, and 3D, from the rotating-wave approximation to the ultrastrong coupling regimes.

As an outlook, let us mention that when several qubits are placed at the positions that form the qubit-photon corner states they are effectively decoupled from environment but still can interact coherently through the overlap of their photonic component. This opens new opportunities to design decoherencefree quantum gates $[39,40]$ in higher dimensions.

\section{ACKNOWLEDGMENTS}

J.J.G.-R. and A.G.-T. acknowledge support from Project PGC2018-094792-B-I00 (MCIU/AEI/FEDER, UE), CSIC Research Platform PTI-001, and CAM/FEDER Project No. S2018/TCS-4342 (QUITEMAD-CM). A.E.F. acknowledges the U.S. Department of Energy, Office of Basic Energy Sciences, for support under Grant No. DE-SC0019275.
[1] E. M. Purcell, Nature (London) 178, 1449 (1956).

[2] K. Drexhage, J. Lumin. 1, 693 (1970).

[3] R. J. Cook and P. W. Milonni, Phys. Rev. A 35, 5081 (1987).

[4] J. Eschner, C. Raab, F. Schmidt-Kaler, and R. Blatt, Nature (London) 413, 495 (2001).

[5] R. H. Lehmberg, Phys. Rev. A 2, 883 (1970).

[6] R. H. Lehmberg, Phys. Rev. A 2, 889 (1970).

[7] U. Dorner and P. Zoller, Phys. Rev. A 66, 023816 (2002).

[8] A. Beige, J. Pachos, and H. Walther, Phys. Rev. A 66, 063801 (2002).

[9] P. Bushev, A. Wilson, J. Eschner, C. Raab, F. Schmidt-Kaler, C. Becher, and R. Blatt, Phys. Rev. Lett. 92, 223602 (2004).

[10] F. Dubin, D. Rotter, M. Mukherjee, C. Russo, J. Eschner, and R. Blatt, Phys. Rev. Lett. 98, 183003 (2007).

[11] A. W. Glaetzle, K. Hammerer, A. Daley, R. Blatt, and P. Zoller, Opt. Commun. 283, 758 (2010).

[12] E. Vetsch, D. Reitz, G. Sagué, R. Schmidt, S. T. Dawkins, and A. Rauschenbeutel, Phys. Rev. Lett. 104, 203603 (2010).

[13] J. D. Thompson, T. G. Tiecke, N. P. de Leon, J. Feist, A. V. Akimov, M. Gullans, A. S. Zibrov, V. Vuletic, and M. D. Lukin, Science 340, 1202 (2013).

[14] A. Goban, C.-L. Hung, S.-P. Yu, J. Hood, J. Muniz, J. Lee, M. Martin, A. McClung, K. Choi, D. Chang, O. Painter, and H. Kimblemblrm, Nat. Commun. 5, 3808 (2014).

[15] J.-B. Béguin, E. M. Bookjans, S. L. Christensen, H. L. Sørensen, J. H. Müller, E. S. Polzik, and J. Appel, Phys. Rev. Lett. 113, 263603 (2014).

[16] P. Solano, P. Barberis-Blostein, F. K. Fatemi, L. A. Orozco, and S. L. Rolston, Nat. Commun. 8, 1857 (2017).

[17] P. Lodahl, S. Mahmoodian, and S. Stobbe, Rev. Mod. Phys. 87, 347 (2015)

[18] X. Gu, S. Chen, and Y.-x. Liu, arXiv:1711.06829.

[19] J.-T. Shen and S. Fan, Phys. Rev. Lett. 95, 213001 (2005).

[20] G. Ordonez, K. Na, and S. Kim, Phys. Rev. A 73, 022113 (2006).

[21] S. Longhi, Eur. Phys. J. B 57, 45 (2007).

[22] S. Tanaka, S. Garmon, and T. Petrosky, Phys. Rev. B 73, 115340 (2006).

[23] L. Zhou, H. Dong, Y.-x. Liu, C. P. Sun, and F. Nori, Phys. Rev. A 78, 063827 (2008).

[24] A. Gonzalez-Tudela, D. Martin-Cano, E. Moreno, L. MartinMoreno, C. Tejedor, and F. J. Garcia-Vidal, Phys. Rev. Lett. 106, 020501 (2011).

[25] C. Gonzalez-Ballestero, F. J. García-Vidal, and E. Moreno, New J. Phys. 15, 073015 (2013).

[26] P. Facchi, M. S. Kim, S. Pascazio, F. V. Pepe, D. Pomarico, and T. Tufarelli, Phys. Rev. A 94, 043839 (2016).

[27] P. Facchi, S. Pascazio, F. V. Pepe, and K. Yuasa, J. Phys. Commun. 2, 035006 (2018).
[28] H. Dong, Z. R. Gong, H. Ian, L. Zhou, and C. P. Sun, Phys. Rev. A 79, 063847 (2009).

[29] T. Tufarelli, F. Ciccarello, and M. S. Kim, Phys. Rev. A 87, 013820 (2013).

[30] T. Tufarelli, M. S. Kim, and F. Ciccarello, Phys. Rev. A 90, 012113 (2014).

[31] I.-C. Hoi, A. Kockum, L. Tornberg, A. Pourkabirian, G. Johansson, P. Delsing, and C. Wilson, Nat. Phys. 11, 1045 (2015).

[32] H. Pichler, S. Choi, P. Zoller, and M. D. Lukin, Proc. Natl. Acad. Sci. USA 114, 11362 (2017).

[33] G. Calajó, Yao-Lung L. Fang, H. U. Baranger, and F. Ciccarello, Phys. Rev. Lett. 122, 073601 (2019).

[34] J. von Neuman and E. P. Wigner, Phys. Z 30, 465 (1929).

[35] C. W. Hsu, B. Zhen, J. Lee, S.-L. Chua, S. G. Johnson, J. D. Joannopoulos, and M. Soljačić, Nature (London) 499, 188 (2013).

[36] R. Parker, J. Sound Vib. 4, 62 (1966).

[37] R. Parker, J. Sound Vib. 5, 330 (1967).

[38] C. W. Hsu, B. Zhen, A. D. Stone, J. D. Joannopoulos, and M. Soljačić, Nat. Rev. Mater. 1, 16048 (2016).

[39] V. Paulisch, H. Kimble, and A. González-Tudela, New J. Phys. 18, 043041 (2016).

[40] A. F. Kockum, G. Johansson, and F. Nori, Phys. Rev. Lett. 120, 140404 (2018).

[41] Yao-Lung L. Fang and H. U. Baranger, Phys. Rev. A 96, 013842 (2017).

[42] C. Müller, J. Combes, A. R. Hamann, A. Fedorov, and T. M. Stace, Phys. Rev. A 96, 053817 (2017).

[43] A. Rosario Hamann, C. Müller, M. Jerger, M. Zanner, J. Combes, M. Pletyukhov, M. Weides, T. M. Stace, and A. Fedorov, Phys. Rev. Lett. 121, 123601 (2018).

[44] A. González-Tudela and J. I. Cirac, Phys. Rev. Lett. 119, 143602 (2017).

[45] A. González-Tudela and J. I. Cirac, Phys. Rev. A 96, 043811 (2017).

[46] F. Galve, A. Mandarino, M. G. Paris, C. Benedetti, and R. Zambrini, Sci. Rep. 7, 42050 (2017).

[47] A. González-Tudela and J. I. Cirac, Quantum 2, 97 (2018).

[48] W. A. Benalcazar, B. A. Bernevig, and T. L. Hughes, Science 357, 61 (2017).

[49] M. Serra-Garcia, V. Peri, R. Süsstrunk, O. R. Bilal, T. Larsen, L. G. Villanueva, and S. D. Huber, Nature (Nature) 555, 342 (2018).

[50] S. Imhof, C. Berger, F. Bayer, J. Brehm, L. W. Molenkamp, T. Kiessling, F. Schindler, C. H. Lee, M. Greiter, T. Neupert et al., Nat. Phys. 14, 925 (2018).

[51] C. W. Peterson, W. A. Benalcazar, T. L. Hughes, and G. Bahl, Nature (London) 555, 346 (2018). 
[52] A. El Hassan, F. K. Kunst, A. Moritz, G. Andler, E. J. Bergholtz, and M. Bourennane, Nat. Photon. 13, 697 (2019).

[53] B.-Y. Xie, G.-X. Su, H.-F. Wang, H. Su, X.-P. Shen, P. Zhan, M.-H. Lu, Z.-L. Wang, and Y.-F. Chen, Phys. Rev. Lett. 122, 233903 (2019).

[54] S. Mittal, V. V. Orre, G. Zhu, M. A. Gorlach, A. Poddubny, and M. Hafezi, Nat. Photon. 13, 692 (2019).

[55] Y. Ota, F. Liu, R. Katsumi, K. Watanabe, K. Wakabayashi, Y. Arakawa, and S. Iwamoto, Optica 6, 786 (2019).

[56] J. J. G. Ripoll, “juanjosegarciaripoll/seeq: First release” (2019).

[57] T. Shi, Y. Chang, and J. J. García-Ripoll, Phys. Rev. Lett. 120, 153602 (2018).

[58] S. R. White and A. E. Feiguin, Phys. Rev. Lett. 93, 076401 (2004).
[59] A. J. Daley, C. Kollath, U. Schollwöck, and G. Vidal, J. Stat. Mech. (2004) P04005.

[60] A. E. Feiguin, in Strongly Correlated Systems (Springer, New York, 2013), pp. 131-152.

[61] S. Paeckel, T. Köhler, A. Swoboda, S. R. Manmana, U. Schollwöck, and C. Hubig, Ann. Phys. 411, 167998 (2019).

[62] A. W. Chin, A. Rivas, S. F. Huelga, and M. B. Plenio, J. Math. Phys. 51, 092109 (2010).

[63] C. A. Büsser, G. B. Martins, and A. E. Feiguin, Phys. Rev. B 88, 245113 (2013).

[64] A. Allerdt and A. E. Feiguin, Front. Phys. 7, 67 (2019).

[65] V. Paulisch, T. Shi, and J. J. Garcia-Ripoll, arXiv:1810.08439. 